\title{
POSITIVITY DETERMINES THE QUANTUM COHOMOLOGY OF GRASSMANNIANS
}

\author{
ANDERS S. BUCH AND CHENGXI WANG
}

\begin{abstract}
We prove that if $X$ is a Grassmannian of type A, then the Schubert basis of the (small) quantum cohomology ring $\mathrm{QH}(X)$ is the only homogeneous deformation of the Schubert basis of the ordinary cohomology ring $H^{*}(X)$ that multiplies with non-negative structure constants. This implies that the (three point, genus zero) Gromov-Witten invariants of $X$ are uniquely determined by Witten's presentation of $\mathrm{QH}(X)$ and the fact that they are non-negative. We conjecture that the same is true for any flag variety $X=G / P$ of simply laced Lie type. For the variety of complete flags in $\mathbb{C}^{n}$, this conjecture is equivalent to Fomin, Gelfand, and Postnikov's conjecture that the quantum Schubert polynomials of type A are uniquely determined by positivity properties. Our proof for Grassmannians answers a question of Fulton.
\end{abstract}

\section{INTRODUCTION}

In this paper we give a proof of Bertram's structure theorems [4] for the (small) quantum cohomology ring of a Grassmannian that uses only that this ring is a graded and associative deformation of the singular cohomology ring [51,38], satisfies Witten's presentation [54], and the fact that the defining Gromov-Witten invariants are non-negative. We expect that similar results hold more generally, so we will start by discussing the quantum cohomology of flag manifolds in general.

Let $X=G / P$ be a flag variety defined by a complex semisimple linear algebraic group $G$ and a parabolic subgroup $P$. Fix a maximal torus $T$ and a Borel subgroup $B$ such that $T \subset B \subset P \subset G$. The opposite Borel subgroup $B^{-} \subset G$ is defined by $B \cap B^{-}=T$. Let $W=N_{G}(T) / T$ be the Weyl group of $G, W_{P}=N_{P}(T) / T$ the Weyl group of $P$, and let $W^{P} \subset W$ be the subset of minimal representatives of the cosets in $W / W_{P}$. Each element $w \in W^{P}$ determines a $B$-stable Schubert variety $X_{w}=\overline{B w \cdot P}$ and an (opposite) $B^{-}$-stable Schubert variety $X^{w}=\overline{B^{-} w \cdot P}$, such that $\operatorname{dim}\left(X_{w}\right)=\operatorname{codim}\left(X^{w}, X\right)=\ell(w)$. The Schubert classes $\left[X^{w}\right]$ form a basis of the cohomology ring $H^{*}(X, \mathbb{Z})$.

Let $\Phi$ be the root system of $G$, with positive roots $\Phi^{+}$and simple roots $\Delta \subset \Phi^{+}$. The parabolic subgroup $P$ is determined by the subset $\Delta_{P}=\left\{\beta \in \Delta \mid s_{\beta} \in W_{P}\right\}$. The group $H_{2}(X, \mathbb{Z})$ has a basis consisting of curve classes $\left[X_{s_{\beta}}\right]$ indexed by the simple roots $\beta \in \Delta \backslash \Delta_{P}$. The elements of $H_{2}(X, \mathbb{Z})$ will be called degrees, and a degree $d=\sum_{\beta} d_{\beta}\left[X_{s_{\beta}}\right]$ is effective, written $d \geq 0$, if $d_{\beta} \geq 0$ for each $\beta$.

Given an effective degree $d \in H_{2}(X, \mathbb{Z})$, we let $\overline{\mathcal{M}}_{0,3}(X, d)$ denote the Kontsevich moduli space of 3-pointed stable maps $f: C \rightarrow X$ of genus zero and degree $d$. See

Date: May 14, 2019.

2010 Mathematics Subject Classification. Primary 14N35; Secondary 14M15, 05E05, 14N15.

The authors were supported in part by NSF grant DMS-1503662. 
[25] for a construction of this moduli space. The dimension is given by

$$
\operatorname{dim} \overline{\mathcal{M}}_{0,3}(X, d)=\operatorname{dim} X+\int_{d} c_{1}\left(T_{X}\right) .
$$

Let $\mathrm{ev}_{i}: \overline{\mathcal{M}}_{0,3}(X, d) \rightarrow X$ be the evaluation map that sends a stable map $f$ to the image of the $i$-th marked point in its domain, for $1 \leq i \leq 3$. Given $u, v, w \in W^{P}$ and $d \in H_{2}(X, Z)$ effective, the corresponding (3 point, genus zero) Gromov-Witten invariant of $X$ is defined by

$$
\left\langle\left[X^{u}\right],\left[X^{v}\right],\left[X_{w}\right]\right\rangle_{d}=\int_{\overline{\mathcal{M}}_{0,3}(X, d)} \operatorname{ev}_{1}^{*}\left[X^{u}\right] \cdot \operatorname{ev}_{2}^{*}\left[X^{v}\right] \cdot \operatorname{ev}_{3}^{*}\left[X_{w}\right] .
$$

This invariant is non-zero only if $\ell(u)+\ell(v)=\ell(w)+\int_{d} c_{1}\left(T_{X}\right)$, in which case it is the number of parametrized curves $\mathbb{P}^{1} \rightarrow X$ of degree $d$ that map three points in $\mathbb{P}^{1}$ to fixed general translates of the Schubert varieties $X^{u}, X^{v}$, and $X_{w}$ (see [25]).

To simplify the statements of our results and conjectures, we will work with quantum cohomology over the field of rational numbers. Let $\mathbb{Q}[q]=\mathbb{Q}\left[q_{\beta}: \beta \in \Delta \backslash\right.$ $\Delta_{P}$ ] denote a polynomial ring in one variable $q_{\beta}$ for each Schubert curve $X_{s_{\beta}}$. These variables are called deformation parameters. We equip this ring with the grading defined by $\operatorname{deg}\left(q_{\beta}\right)=\int_{X_{s_{\beta}}} c_{1}\left(T_{X}\right)$. Given an effective degree $d=\sum_{\beta} d_{\beta}\left[X_{s_{\beta}}\right]$ in $H_{2}(X, \mathbb{Z})$, set $q^{d}=\prod q_{\beta}^{d_{\beta}}$. The (small) quantum cohomology ring $\mathrm{QH}(X)$ is a graded $\mathbb{Q}[q]$-algebra, which as a $\mathbb{Q}[q]$-module is defined by $\mathrm{QH}(X)=H^{*}(X, \mathbb{Q}) \otimes \mathbb{Q} \mathbb{Q}[q]$. In other words, the Schubert classes $\left[X^{w}\right]$ for $w \in W^{P}$ form a $\mathbb{Q}[q]$-basis of this ring. The grading is given by $\operatorname{deg}\left[X^{w}\right]=\ell(w)$, and the multiplicative structure is defined by

$$
\left[X^{u}\right] \star\left[X^{v}\right]=\sum_{w, d \geq 0}\left\langle\left[X^{u}\right],\left[X^{v}\right],\left[X_{w}\right]\right\rangle_{d} q^{d}\left[X^{w}\right]
$$

where the sum is over all $w \in W^{P}$ and effective $d \in H_{2}(X, \mathbb{Z})$. It was proved by Ruan and Tian [51] and by Kontsevich and Manin [38] that this product is associative.

The definition of the quantum cohomology ring came from physics, see [54]. In mathematics the quantum ring $\mathrm{QH}(X)$ provides an effective tool for computing the Gromov-Witten invariants of $X$, as these invariants can be calculated if enough is known about the structure of the ring $\mathrm{QH}(X)$. For example, the computation of Gromov-Witten invariants is reduced to combinatorics if one knows all invariants $\left\langle\left[X^{u}\right],\left[X^{v}\right],\left[X_{w}\right]\right\rangle_{d}$ for which $\left[X^{u}\right]$ belongs to a set of generators of the cohomology ring $H^{*}(X, \mathbb{Q})$. Formulas for Gromov-Witten invariants of this type are frequently called quantum Pieri formulas or quantum Chevalley formulas, see [4, 22, 21, 26, $39,40,49,14,31]$ for examples.

It is in general not enough to know a presentation of the ring $\mathrm{QH}(X)$ by generators and relations for the Gromov-Witten invariants of $X$ to be determined (see Example 6). However, a presentation together with a quantum Giambelli formula that expresses each Schubert class $\left[X^{w}\right]$ as a polynomial in the chosen generators of $\mathrm{QH}(X)$ is sufficient. Examples of presentations and Giambelli formulas in quantum cohomology can be found in $[54,53,4,28,35,20,37,22,39,40,50,16,15,1]$.

It should be noted that quantum cohomology is not functorial, so there is in general no ring homomorphism $\mathrm{QH}(G / P) \rightarrow \mathrm{QH}(G / B)$ that extends the pullback map $H^{*}(G / P, \mathbb{Q}) \rightarrow H^{*}(G / B, \mathbb{Q})$ along the projection $G / B \rightarrow G / P$. For this reason, the study of quantum cohomology must be carried out separately for each 
flag variety $X$. Relations between the Gromov-Witten invariants of $G / B$ and $G / P$ do exist in the form of the Peterson comparison formula [55], but it requires work to extract algebraic properties of the quantum ring from this formula (see e.g. [31]).

The first examples of structure theorems for the quantum cohomology ring $\mathrm{QH}(X)$ were provided by Witten [54] and Bertram [4] when $X=\operatorname{Gr}(m, n)$ is the Grassmannian of $m$-planes in $\mathbb{C}^{n}$. Bertram's results, a Pieri formula and a Giambelli formula for $\mathrm{QH}(X)$, were proved by applying results from intersection theory to the quot scheme compactification of the moduli space $\mathcal{M}_{0,3}(X, d)$ of parametrized curves. More elementary proofs were later given in [6] by studying the kernels and spans of the curves counted by Gromov-Witten invariants. The idea is that if a Gromov-Witten invariant of a Grassmannian is non-zero, then the span of any counted curve, defined as the linear span of the $m$-planes given by points of the curve, will produce a point in a related intersection of Schubert varieties in a different Grassmannian, and this intersection can be studied with classical Schubert calculus. More generally, it was shown in [13] that the map that sends a curve to its kernel-span pair provides a bijection between the curves counted by a Gromov-Witten invariant with the points in an intersection of Schubert varieties in a two-step flag variety. Further generalizations of this quantum equals classical phenomenon can be found in $[18,14,17,10]$ and the references therein.

The quantum cohomology ring of the variety $X=\mathrm{GL}(n) / B$ of complete flags in $\mathbb{C}^{n}$ is described by structure theorems of Fomin, Gelfand, and Postnikov [22]. The main result of [22] states that the quantum Schubert polynomials provide Giambelli formulas for the Schubert classes in $\mathrm{QH}(X)$. In addition to a known presentation of the ring $\mathrm{QH}(X)[28,35,20]$ and a special case of the Giambelli formula [20], the proof uses the geometric fact that the structure constants of $\mathrm{QH}(X)$ are non-negative integers. Moreover, it was conjectured in [22] that the quantum Schubert polynomials are uniquely determined by the non-negativity of their structure constants, in addition to the presentation, grading, and deformation properties of $\mathrm{QH}(X)$. Positivity properties have received much attention in the study of quantum cohomology $[48,2,12,7]$, but usually as a benchmark of our understanding of the combinatorial aspects of quantum cohomology rather than a tool for proving other results.

Fulton asked the related question, whether the quantum cohomology ring of a Grassmannian is uniquely determined by positivity conditions [24]. The immediate answer is no; it is always possible to rescale the deformation parameter $q$ by a positive factor $\alpha>0$, or equivalently, multiply each Gromov-Witten invariant $\left\langle\left[X^{u}\right],\left[X^{v}\right],\left[X_{w}\right]\right\rangle_{d}$ by $\alpha^{-d}$. However, this modification will change the relations among the Schubert class generators of $\mathrm{QH}(X)$. In this paper we show that, for Grassmannians, rescaling the Gromov-Witten invariants is the only change to the quantum cohomology ring that preserves its formal properties as well as positivity of its structure constants (Corollary 19). By adding the condition that the quantum cohomology ring satisfies Witten's presentation [54], this ring is uniquely determined, and Bertram's structure theorems can be proved with purely combinatorial methods.

Our results establish the Grassmannian case of the following conjecture, which also generalizes the conjectured uniqueness of quantum Schubert polynomials [22]. 
Conjecture 1. Let $X=G / P$ be any flag variety of simply laced Lie type. Then the Schubert basis of $\mathrm{QH}(X)$ is the only homogeneous $\mathbb{Q}[q]$-basis that deforms the Schubert basis of $H^{*}(X, \mathbb{Q})$ and multiplies with non-negative structure constants.

In other words, if $\left\{\tau_{w} \mid w \in W^{P}\right\}$ is any $\mathbb{Q}[q]$-basis of $\mathrm{QH}(X)$ such that $\tau_{w}$ is homogeneous of degree $\ell(w), \tau_{w}-\left[X^{w}\right]$ belongs to the ideal $\langle q\rangle$ generated by the deformation parameters $q_{\beta}$, and each product $\tau_{u} \star \tau_{v}$ is a non-negative linear combination of the $\mathbb{Q}$-basis $\left\{q^{d} \tau_{w}\right\}$, then we have $\tau_{w}=\left[X^{w}\right]$ for all $w \in W^{P}$.

In addition to Grassmannians of type $A$, we have verified Conjecture 1 when $X=\mathrm{OG}(n, 2 n)$ is a maximal orthogonal Grassmannian of type $\mathrm{D}_{n}$, with $n \leq 6$, and when $X=\operatorname{OG}(1,2 n)$ is any quadric hypersurface of even dimension. The condition that the root system of $G$ is simply laced is necessary, since the conjecture is false for the Lagrangian Grassmannian LG $(2,4)$ of type $\mathrm{C}_{2}$, a 3-dimensional quadric. These examples are treated in Section 2. We have also obtained computational verification of Conjecture 1 for all partial flag varieties $\mathrm{GL}(n) / P$ of dimension at most 10 , except $\mathrm{Fl}\left(1,2,3 ; \mathbb{C}^{5}\right), \mathrm{Fl}\left(1,2,4 ; \mathbb{C}^{5}\right)$, and $\mathrm{Fl}\left(1,2,3,4 ; \mathbb{C}^{5}\right)$. We note that, since the number of monomials in $\mathbb{Q}[q]$ grows very fast with respect to total degree when $X$ has large Picard rank, so does the computational complexity of this problem.

Our proof of Conjecture 1 for Grassmannians of type A uses that quantum multiplication with the top Chern class of a tautological vector bundle maps any Schubert class to a power of $q$ times a different Schubert class. This follows from Bertram's quantum Pieri formula [4] and is a special case of the Seidel representation of the group $\pi_{1}(\operatorname{Aut}(X))$ on $\mathrm{QH}(X) /\langle q-1\rangle$, which was introduced in [52] and computed explicitly for flag varieties in [19]. Here $\langle q-1\rangle$ is the ideal generated by all differences $q_{\beta}-1$. For any flag variety $X$, the action of this representation is given by quantum multiplication by certain Schubert classes $\left[X^{u}\right]$ of finite order in the group of units $(\mathrm{QH}(X) /\langle q-1\rangle)^{\times}$. We show that basis elements of finite order in any non-negative quantum deformation must behave in a similar way (Lemma 3), which gives a way to compare different basis elements. For the Grassmannian $X=\operatorname{Gr}(m, n)$ we then observe that the sum of the degrees of the Schubert classes in any Seidel orbit is always the same number, namely $\frac{1}{2} m n(n-m)$ (Lemma 16). This is a special phenomenon for Grassmannians of type A; a counterexample for the variety of complete flags in $\mathbb{C}^{6}$ is provided in Example 21. The Grassmannian case of Conjecture 1 is proved by combining these observations.

The quantum cohomology ring of a flag variety is generalized by the quantum $K$-theory ring $[27,42]$, which has received much attention in recent years, see e.g. $[17,9,29,32,8,41,33,34,3,11]$. In contrast to quantum cohomology, structure theorems for the quantum $K$-theory ring are known only for cominuscule spaces $[17,29,8]$, while conjectures exist in other cases [43, 44]. It would be very interesting to find a way to uniquely describe the quantum $K$-theory ring in terms of combinatorial properties. The same question can be asked about the equivariant quantum cohomology ring [36, 37, 49, 48, 2].

Another interesting open problem is to give a combinatorial proof that the quantum cohomology ring of a Grassmannian, as defined by Bertram's structure theorems [4], has non-negative structure constants (see [5]). While positive formulas for these Gromov-Witten invariants do exist [12, 7], their proofs utilize the quantum equals classical theorem $[13,17]$ rather than studying the quantum cohomology ring directly. The methods presented in this paper do not solve this problem; we 
simply prove that any quantum deformation of a Grassmannian with non-negative structure constants must obey Bertram's structure theorems.

This paper is organized as follows. In Section 2 we formulate the notion of a quantum deformation, which captures the properties of the quantum cohomology ring that are automatic from its definition. We also provide some initial examples of Conjecture 1. In Section 3 we prove that any non-negative quantum deformation of a Grassmannian is obtained from the quantum cohomology ring by rescaling the defining Gromov-Witten invariants. This section can also be read as a combinatorial introduction to the quantum cohomology of Grassmannians. We thank W. Fulton for his inspiring question.

\section{QuAntum DeFormations}

2.1. Quantum deformations. The (small) quantum cohomology ring $\mathrm{QH}(X)$ of a flag variety $X=G / P$ is a quantum deformation of the singular cohomology ring $H^{*}(X, \mathbb{Q})$ in the following sense.

Definition 2. A quantum deformation of $H^{*}(X, \mathbb{Q})$ is a graded associative $\mathbb{Q}[q]$ algebra $\mathrm{QH}$ with unit, together with a $\mathbb{Q}[q]$-basis $\left\{\tau_{w}: w \in W^{P}\right\}$ of $\mathrm{QH}$ indexed by $W^{P}$, such that each basis element $\tau_{w}$ is homogeneous of degree $\ell(w)$, and the assignments $\tau_{w} \mapsto\left[X^{w}\right]$ and $q_{\beta} \mapsto 0$ define a ring homomorphism $\mathrm{QH} \rightarrow H^{*}(X, \mathbb{Q})$.

We will often denote a quantum deformation $\left(\mathrm{QH},\left\{\tau_{w}\right\}\right)$ simply by $\mathrm{QH}$, and we will occasionally misuse terminology and call it a quantum deformation of $X$. Notice that $\mathrm{QH} /\langle q\rangle \cong H^{*}(X, \mathbb{Q})$, where $\langle q\rangle \subset \mathrm{QH}$ is the ideal generated by the deformation parameters $q_{\beta}$. In addition, the set $\mathcal{T}=\left\{q^{d} \tau_{w} \mid w \in W^{P}, d \geq 0\right\}$ is a basis of $\mathrm{QH}$ as a vector space over $\mathbb{Q}$. The structure constants of $\mathrm{QH}$ with respect to this basis are the numbers $N_{u, v}^{w, d} \in \mathbb{Q}$, indexed by $u, v, w \in W^{P}$ and $d \geq 0$, defined by the identity

$$
\tau_{u} \tau_{v}=\sum_{w, d} N_{u, v}^{w, d} q^{d} \tau_{w}
$$

We will say that the quantum deformation $\mathrm{QH}$ is non-negative if $N_{u, v}^{w, d} \geq 0$ for all $u, v, w \in W^{P}$ and $d \geq 0$.

The quantum deformation of main interest is the quantum cohomology ring $\mathrm{QH}(X)$, equipped with its basis of Schubert classes. Since the Gromov-Witten invariants of $X$ are enumerative [25], we know a priori that $\mathrm{QH}(X)$ is a non-negative quantum deformation. It is natural to ask which non-negative quantum deformations exist.

One way to produce new quantum deformations is to replace the Schubert basis of $\mathrm{QH}(X)$ with any $\mathbb{Q}[q]$-basis $\left\{\tau_{w}: w \in W^{P}\right\}$ for which each element $\tau_{w}$ is a homogeneous deformation of the Schubert class $\left[X^{w}\right]$, i.e. $\tau_{w}-\left[X^{w}\right] \in\langle q\rangle$. Such deformations will be called change-of-basis quantum deformations of $H^{*}(X, \mathbb{Q})$.

We remark that historically, a presentation of the quantum ring $\mathrm{QH}(X)$ was known early on for many flag varieties [54, 28, 20,37]. For example, Witten's paper [54] contains a presentation of $\mathrm{QH}(X)$ when $X$ is a Grassmannian (see also [53]). When a presentation is known, a determination of the quantum ring $\mathrm{QH}(X)$ and the associated Gromov-Witten invariants of $X$ is equivalent to finding the correct non-negative change-of-basis quantum deformation of $X$, so it is also natural to ask for a classification of these deformations. Conjecture 1 states that, if $X$ is 
of simply laced Lie type, then $\mathrm{QH}(X)$ is the only non-negative change-of-basis quantum deformation up to isomorphism. ${ }^{1}$

2.2. General properties. Let $\left(\mathrm{QH},\left\{\tau_{w}\right\}\right)$ be a quantum deformation of $H^{*}(X, \mathbb{Q})$ and let $\phi: \mathrm{QH} \rightarrow H^{*}(X, \mathbb{Q})$ be the ring homomorphism of Definition 2. Assume that $\phi(\eta)=\left[X^{u}\right]$ for some $\eta \in \mathrm{QH}$ and $u \in W^{P}$. Then $\eta-\tau_{u} \in\langle q\rangle$. If $\eta$ is homogeneous with $\operatorname{deg}(\eta)<\operatorname{deg}\left(q_{\beta}\right)$ for all $\beta \in \Delta \backslash \Delta_{P}$, then this implies that $\eta=\tau_{u}$. For example we have $\tau_{e}=1$, where $e \in W$ is the identity element.

Lemma 3. Let $\left(\mathrm{QH},\left\{\tau_{w}\right\}\right)$ be any non-negative quantum deformation of $H^{*}(X, \mathbb{Q})$ and let $u_{1}, u_{2}, \ldots, u_{\ell} \in W^{P}$. Assume that the product $\tau_{u_{\ell}} \tau_{u_{\ell-1}} \cdots \tau_{u_{1}}$ is a non-zero multiple of a monomial from $\mathbb{Q}[q]$, that is, $\tau_{u_{\ell}} \tau_{u_{\ell-1}} \cdots \tau_{u_{1}}=c q^{d}$ for some rational number $c>0$ and effective degree $d$. (1) For every $v \in W^{P}$, the product $\tau_{u_{1}} \tau_{v}$ is a positive multiple of a single element from $\mathcal{T}=\left\{q^{d} \tau_{w}\right\}$. (2) If $\left[X^{u_{1}}\right] \cdot\left[X^{v}\right]=\left[X^{w}\right]$ holds in $H^{*}(X, \mathbb{Q})$, then $\tau_{u_{1}} \tau_{v}=\tau_{w}$ holds in $\mathrm{QH}$.

Proof. If part (1) is false, then since $\tau_{u_{\ell}} \cdots \tau_{u_{1}} \tau_{v}=c q^{d} \tau_{v}$, we can choose $p>0$ such that the expansion of $\tau_{u_{p}} \cdots \tau_{u_{1}} \tau_{v}$ involves more than one basis element from $\mathcal{T}$, whereas $\tau_{u_{p+1}} \tau_{u_{p}} \cdots \tau_{u_{1}} \tau_{v}$ is a multiple of a single basis element $q^{e} \tau_{w}$. Write $\tau_{u_{p}} \cdots \tau_{u_{1}} \tau_{v}=b_{1} q^{d_{1}} \tau_{v_{1}}+\cdots+b_{r} q^{d_{r}} \tau_{v_{r}}$ where $r \geq 2$ and $b_{i}>0$ for $1 \leq i \leq r$. Since $\tau_{u_{p+1}} q^{d_{i}} \tau_{v_{i}}$ is a non-negative linear combination of $\mathcal{T}$ for each $i$, and since $\tau_{u_{p+1}} \sum b_{i} q^{d_{i}} \tau_{v_{i}}$ is a multiple of $q^{e} \tau_{w}$, we deduce that $\tau_{u_{p+1}} q^{d_{i}} \tau_{v_{i}}$ is a multiple of $q^{e} \tau_{w}$ for each $i$. This contradicts that multiplication by $\tau_{u_{p+1}}$ is an injective operation on QH. Finally, part (2) follows from part (1).

2.3. Examples. We finish this section by examining Conjecture 1 for a selection of cominuscule spaces $X=G / P$. The quantum cohomology rings these varieties contain only one deformation parameter which we denote by $q$.

Example 4. Let $X=\mathrm{OG}(1,2 n)$ be the quadric hypersurface of dimension $2 n-2$. A description of the Schubert classes and quantum cohomology ring of this variety can be found in $[18,14]$. Let $\left\{\tau_{w}: w \in W^{P}\right\}$ be any homogeneous deformation of the Schubert basis of $\mathrm{QH}(X)$ that multiplies with non-negative structure constants. Since $\operatorname{deg}(q)=\operatorname{dim}(X)=2 n-2$, it follows that $\tau_{w}=\left[X^{w}\right]$ for all $w \in W^{P}$, except for the element $w_{0}^{P}$ representing a point. Let $u \in W^{P}$ have length $n-1$, and choose $u^{\vee} \in W^{P}$ such that $\left[X^{u}\right] \cdot\left[X^{u^{\vee}}\right]=\left[X^{w_{0}^{P}}\right]$ in $H^{*}(X ; \mathbb{Q})$. It follows from [19, Thm. 1] or $\left[14\right.$, Thm. 3.4] that $\tau_{u}^{4}=\left[X^{u}\right]^{4}=q^{2}$, so we deduce from Lemma 3 that $\tau_{w_{0}^{P}}=\tau_{u} \star \tau_{u^{\vee}}=\left[X^{u}\right] \star\left[X^{u^{\vee}}\right]=\left[X^{w_{0}^{P}}\right]$. This shows that Conjecture 1 holds for $X$. Similar arguments can be used to show that any non-negative quantum deformation of $X$ is obtained from $\mathrm{QH}(X)$ by rescaling the defining Gromov-Witten invariants.

Example 5. Let $X=\mathrm{OG}(n, 2 n)$ be the maximal orthogonal Grassmannian of type $\mathrm{D}_{n}$. The Schubert classes and quantum cohomology of this space are described in [40]. We have $\operatorname{dim}(X)=\left(\begin{array}{l}n \\ 2\end{array}\right)$ and $\operatorname{deg}(q)=2 n-2$. The elements of $W^{P}$ can be identified with strict partitions $\lambda=\left(\lambda_{1}>\lambda_{2}>\cdots>\lambda_{\ell}>0\right)$ with $\lambda_{1} \leq n-1$, so that $\operatorname{codim}\left(X^{\lambda}, X\right)=|\lambda|=\sum \lambda_{i}$. Let $\left\{\tau_{\lambda}\right\}$ be any homogeneous deformation of the Schubert basis of $\mathrm{QH}(X)$ that multiplies with non-negative structure constants. Then we have $\tau_{\lambda}=\left[X^{\lambda}\right]$ for $|\lambda|<2 n-2$. The classical Pieri formula [30] implies that $\left[X^{n-1}\right] \cdot\left[X^{\lambda}\right]=\left[X^{n-1, \lambda}\right]$ holds in $H^{*}(X, \mathbb{Q})$ whenever $\lambda_{1}<n-1$, and [40,

\footnotetext{
${ }^{1} \mathrm{An}$ (iso)morphism $\left(\mathrm{QH},\left\{\tau_{w}\right\}\right) \rightarrow\left(\mathrm{QH}^{\prime},\left\{\tau_{w}^{\prime}\right\}\right)$ of quantum deformations is a $\mathbb{Q}[q]$-algebra homomorphism that maps $\tau_{w}$ to $\tau_{w}^{\prime}$ for each $w \in W^{P}$.
} 
Cor. 5] gives $\tau_{n-1}^{2}=\left[X^{n-1}\right]^{2}=q$. Lemma 3 therefore implies that $\tau_{n-1, \lambda}=$ $\tau_{n-1} \star \tau_{\lambda}=\left[X^{n-1}\right] \star\left[X^{\lambda}\right]=\left[X^{n-1, \lambda}\right]$ for all strict partitions $\lambda$ such that $\lambda_{1}<n-1$ and $|\lambda|<2 n-2$. This proves Conjecture 1 when $n \leq 5$, and for $n=6$ we obtain $\tau_{\lambda}=\left[X^{\lambda}\right]$ for all strict partitions except $\nu=(4,3,2,1)$ and $(5, \nu)$. We must have $\tau_{\nu}=\left[X^{\nu}\right]+c q$ and $\tau_{5, \nu}=\tau_{5} \star \tau_{\nu}=\left[X^{5, \nu}\right]+c q \tau_{5}$ for some constant $c \in \mathbb{Q}$. Since $\tau_{1} \star \tau_{4,3,2}=\left[X^{1}\right] \star\left[X^{4,3,2}\right]=\left[X^{5,3,2}\right]+\left[X^{\nu}\right]=\tau_{5,3,2}+\tau_{\nu}-c q$, it follows that $c \leq 0$. Since $\tau_{1} \star \tau_{\nu}=\left[X^{1}\right] \star\left[X^{\nu}\right]+c q\left[X^{1}\right]=\left[X^{5,3,2,1}\right]+c q\left[X^{1}\right]=\tau_{5,3,2,1}+c q \tau_{1}$, it follows that $c \geq 0$. This shows that Conjecture 1 also holds for $\mathrm{OG}(6,12)$.

The following example shows that Conjecture 1 does not hold for arbitrary flag varieties. It would be interesting to know if the non-negative quantum deformations of any flag variety correspond to points in a rational polyhedral cone.

Example 6. Let $X=\mathrm{LG}(2,4)$ be the Lagrangian Grassmannian of type $\mathrm{C}_{2}$. Then $H^{*}(X, \mathbb{Q})$ has a basis of Schubert classes $\left\{\left[X^{0}\right]=1,\left[X^{1}\right],\left[X^{2}\right],\left[X^{3}\right]=[\mathrm{pt}]\right\}$, where $\left[X^{p}\right] \in H^{2 p}(X)$. The multiplicative structure of $H^{*}(X, \mathbb{Q})$ is determined by $\left[X^{1}\right]^{2}=2\left[X^{2}\right]$ and $\left[X^{1}\right] \cdot\left[X^{2}\right]=\left[X^{3}\right]$. One can check that any quantum deformation $\left(\mathrm{QH},\left\{\tau_{0}=1, \tau_{1}, \tau_{2}, \tau_{3}\right\}\right)$ of $H^{*}(X, \mathbb{Q})$ is given by

$$
\begin{aligned}
\tau_{1}^{2} & =2 \tau_{2} \\
\tau_{1} \tau_{2} & =\tau_{3}+(2 a-b) q \\
\tau_{1} \tau_{3} & =b q \tau_{1} \\
\tau_{2}^{2} & =a q \tau_{1} \\
\tau_{2} \tau_{3} & =b q \tau_{2} \\
\tau_{3}^{2} & =(2 b-2 a) q \tau_{3}+\left(2 a b-b^{2}\right) q^{2}
\end{aligned}
$$

where $a, b \in \mathbb{Q}$ are constants. It is non-negative if and only if $a \leq b \leq 2 a$, and it is a change-of-basis deformation if and only if $a=1$. In particular, we obtain a nonnegative change-of-basis deformation of $H^{*}(X, \mathbb{Q})$ whenever $a=1$ and $1 \leq b \leq 2$. The quantum cohomology ring $\mathrm{QH}(X)$ corresponds to $a=b=1[39]$.

\section{Grassmannians}

Let $X=\operatorname{Gr}(m, n)$ be the Grassmannian of $m$-dimensional vector subspaces in $\mathbb{C}^{n}$ and set $k=n-m$. In this section we classify the non-negative quantum deformations of the cohomology ring $H^{*}(X, \mathbb{Q})$. We start by recalling some basic definitions and facts about symmetric functions.

3.1. Partitions. We will identify any partition $\lambda=\left(\lambda_{1} \geq \lambda_{2} \geq \cdots \geq \lambda_{\ell}>0\right)$ with its Young diagram of boxes, which has $\lambda_{i}$ boxes in row $i$. Rows are numbered from top to bottom and are left aligned. The length $\ell(\lambda)$ is the number of non-empty rows, and the weight $|\lambda|=\sum \lambda_{i}$ is the total number of boxes. We write $\lambda_{i}=0$ for $i>\ell(\lambda)$. The conjugate Young diagram $\lambda^{\prime}$ is obtained by mirroring $\lambda$ in the diagonal, so that rows and columns are interchanged.

Given an additional partition $\mu$, we write $\mu \subset \lambda$ if and only if $\mu_{i} \leq \lambda_{i}$ for each $i$. In this case $\lambda / \mu$ denotes the skew diagram of boxes in $\lambda$ that are not in $\mu$. This skew diagram is a horizontal strip if it has at most one box in each column, and a vertical strip if it has at most one box in each row. The outer rim of a $\lambda$ is the skew diagram of boxes in $\lambda$ that are not strictly north and strictly west of other boxes in $\lambda$. In other words, the box in row $i$ and column $j$ belongs to the outer rim of $\lambda$ if and only if $\lambda_{i+1} \leq j \leq \lambda_{i}$. 
For integers $r, c \geq 0$ we let $\left(c^{r}\right)=(c, c, \ldots, c)$ denote the rectangular Young diagram with $r$ rows and $c$ columns. Since the Schubert varieties in $X=\operatorname{Gr}(m, n)$ can be indexed by partitions $\lambda$ contained in the rectangle $\left(k^{m}\right)$ (see Section 3.3), we also denote this rectangle as $m \times k$.

Recall also that the lexicographic order on integer sequences is defined by $\mu<_{\operatorname{lex}} \lambda$ if and only if for some index $p$ we have $\mu_{i}=\lambda_{i}$ for $1 \leq i<p$ whereas $\mu_{p}<\lambda_{p}$. The sequence $\mu$ is smaller than $\lambda$ in the degree-lexicographic order if and only if $|\mu|<|\lambda|$, or $|\mu|=|\lambda|$ and $\mu<_{\operatorname{lex}} \mu$.

3.2. Symmetric functions. Given a sequence $h=\left(h_{1}, h_{2}, \ldots\right)$ of elements in a commutative ring $R$ and a partition $\lambda=\left(\lambda_{1} \geq \lambda_{2} \geq \cdots \geq \lambda_{\ell} \geq 0\right)$, define

$$
\Delta_{\lambda}(h)=\operatorname{det}\left(h_{\lambda_{i}+j-i}\right)_{\ell \times \ell} .
$$

Here we set $h_{0}=1$ and $h_{i}=0$ for $i<0$. Let $e=\left(e_{1}, e_{2}, \ldots\right)$ be the dual sequence defined by $e_{p}=\Delta_{\left(1^{p}\right)}(h)=\operatorname{det}\left(h_{1+j-i}\right)_{p \times p}$. Equivalently, we can define $e_{p}$ recursively by the formula

$$
e_{p}=\sum_{i=1}^{p}(-1)^{i-1} h_{i} e_{p-i} .
$$

We need the following facts about Schur polynomials. Proofs can be found in e.g. $[45$, Ch. I] or $[23, \S 2.2, \S 6.1]$.

Fact 7. We have $e_{p} \Delta_{\lambda}(h)=\sum_{\mu} \Delta_{\mu}(h)$, where the sum is over all partitions $\mu$ obtained by adding a vertical strip of $p$ boxes to $\lambda$.

Fact 8. We have $\Delta_{\lambda}(e)=\Delta_{\lambda^{\prime}}(h)$, where $\lambda^{\prime}$ is the conjugate partition of $\lambda$.

Define the polynomial ring $S=\mathbb{Q}\left[c_{1}, \ldots, c_{m}\right]$, with the grading defined by $\operatorname{deg}\left(c_{i}\right)=i$. Let $c=\left(c_{1}, c_{2}, \ldots c_{m}, 0,0, \ldots\right)$ be the sequence of its variables, with $c_{i}=0$ for $i>m$. Let $\sigma=\left(\sigma_{1}, \sigma_{2}, \ldots\right)$ be the dual sequence defined by $\sigma_{p}=\Delta_{\left(1^{p}\right)}(c)=\operatorname{det}\left(c_{1+j-i}\right)_{p \times p}$. For each partition $\lambda$ we set $\sigma_{\lambda}=\Delta_{\lambda}(\sigma)$. This is a homogeneous element of $S$ of degree $|\lambda|$.

Lemma 9. The set $\left\{\sigma_{\lambda} \mid \ell(\lambda) \leq m\right\}$ is a $\mathbb{Q}$-basis of $S$, and we have $\sigma_{\lambda}=0$ whenever $\ell(\lambda)>m$.

Proof. By Fact 8 we have $\sigma_{\lambda}=\Delta_{\lambda^{\prime}}(c)$. If $\ell(\lambda)>m$, then all entries in the top row of the determinant defining $\Delta_{\lambda^{\prime}}(c)$ are zero. Otherwise the initial term of $\Delta_{\lambda^{\prime}}(c)$ is $c_{1}^{\lambda_{1}-\lambda_{2}} c_{2}^{\lambda_{2}-\lambda_{3}} \cdots c_{m}^{\lambda_{m}}$, where the monomials $c_{1}^{p_{1}} c_{2}^{p_{2}} \ldots c_{m}^{p_{m}}$ of $S$ are ordered according to the lexicographic order on the exponent vectors $\left(p_{m}, p_{m-1}, \ldots, p_{1}\right)$. The lemma follows from this.

Lemma 10. The set $\left\{\sigma_{\lambda} \mid \lambda \subset m \times k\right\}$ is a $\mathbb{Q}$-basis of the ring $S /\left\langle\sigma_{k+1}, \ldots, \sigma_{n}\right\rangle$.

Proof. By Lemma 9 it is enough to show that $I=\left\langle\sigma_{k+1}, \sigma_{k+2}, \ldots, \sigma_{n}\right\rangle$ has a basis consisting of all elements $\sigma_{\lambda}$ for which $\ell(\lambda) \leq m$ and $\lambda_{1}>k$. Fact 7 implies that $I$ is contained in the span of these basis elements. By using the identity $\sigma_{p}=\sum_{i=1}^{m}(-1)^{i-1} c_{i} \sigma_{p-i}$, it follows by induction on $p$ that $\sigma_{p} \in I$ for all $p>k$. Therefore all elements in the top row of the determinant defining $\sigma_{\lambda}$ are contained in $I$ when $\lambda_{1}>k$.

Corollary 11. The element $\sigma_{n}$ is a non-zero divisor in $S /\left\langle\sigma_{k+1}, \ldots, \sigma_{n-1}\right\rangle$. 
Proof. Set $J=\left\langle\sigma_{k+1}, \ldots, \sigma_{n-1}\right\rangle \subset S$ and $K=\left\{f \in S \mid \sigma_{n} f \in J\right\}$. We must show that $K=J$. Otherwise choose a prime ideal $P \subset S$ such that $(K / J)_{P} \neq 0$. Then $P$ contains $I=\left\langle\sigma_{k+1}, \sigma_{k+2}, \ldots, \sigma_{n}\right\rangle$, and $S_{P} / I_{P}$ has Krull dimension zero by Lemma 10. It follows that the generators of $I$ form a regular sequence in $S_{P}$ [47, Thm. 17.4], which contradicts that $\sigma_{n}$ is a zero-divisor in $S_{P} / J_{P}$.

3.3. Cohomology of Grassmannians. We summarize the main facts about the cohomology of Grassmannians. More details and proofs can be found in e.g. [23, $\S 9.4]$ or $[46, \S 3]$.

Each partition $\lambda=\left(\lambda_{1} \geq \cdots \geq \lambda_{m}\right)$ contained in the rectangle $m \times k$ defines a Schubert variety in $X=\operatorname{Gr}(m, n)$ given by

$$
X^{\lambda}=\left\{V \in X \mid \operatorname{dim}\left(V \cap F^{k+i-\lambda_{i}}\right) \geq i \forall 1 \leq i \leq m\right\},
$$

where $F^{k+i-\lambda_{i}}$ denotes the span of the last $k+i-\lambda_{i}$ coordinate basis vectors in $\mathbb{C}^{n}$. We have $\operatorname{codim}\left(X^{\lambda}, X\right)=|\lambda|$. With the notation of the introduction we have $X=G / P$, where $G=\operatorname{GL}(n, \mathbb{C})$ and $P$ is the stabilizer of the span of the first $m$ basis vectors in $\mathbb{C}^{n}$. If we use the maximal torus $T$ of diagonal matrices and the Borel subgroup $B$ of upper triangular matrices, and identify the Weyl group $W$ with the subgroup of permutation matrices in $G$, then the set $W^{P}$ consists of all permutations $w=\left(w_{1}, w_{2}, \ldots, w_{n}\right)$ with descent only at position $m$, i.e. $w_{i}<w_{i+1}$ for $i \neq m$. We then have $X^{w}=X^{\lambda}$ where $\lambda=\left(w_{m}-m, \ldots, w_{2}-2, w_{1}-1\right)$.

The Schubert classes $\left[X^{\lambda}\right]$ form a basis of $H^{*}(X, \mathbb{Q})$ as a vector space over $\mathbb{Q}$. The special Schubert classes $\left[X^{\left(1^{p}\right)}\right]$ are the Chern classes of the dual of the tautological subbundle $\mathcal{S} \subset X \times \mathbb{C}^{n}$ of rank $m$. The classical Pieri formula states that multiplication with these classes in $H^{*}(X, \mathbb{Q})$ is determined by

$$
\left[X^{\left(1^{p}\right)}\right] \cdot\left[X^{\lambda}\right]=\sum_{\mu}\left[X^{\mu}\right]
$$

where the sum is over all partitions $\mu \subset m \times k$ obtained by adding a vertical strip of $p$ boxes to $\lambda$.

Lemma 12. The linear map $S /\left\langle\sigma_{k+1}, \ldots, \sigma_{n}\right\rangle \rightarrow H^{*}(X, \mathbb{Q})$ defined by $\sigma_{\lambda} \mapsto\left[X^{\lambda}\right]$ is an isomorphism of graded rings.

Proof. Since $c_{p}=\sigma_{\left(1^{p}\right)}$ is mapped to $\left[X^{\left(1^{p}\right)}\right]$, it follows from Fact 7 and the Pieri formula (1) that any product $c_{p} \sigma_{\lambda}$ is mapped to $\left[X^{\left(1^{p}\right)}\right] \cdot\left[X^{\lambda}\right]$. Since $S$ is generated by $c_{1}, \ldots, c_{m}$, this implies that the map is a ring homomorphism.

3.4. Quantum deformations of Grassmannians. The quantum cohomology ring $\mathrm{QH}(X)$ contains a single deformation parameter $q$ of degree $n$. We next give a combinatorial construction of the non-negative quantum deformations of $H^{*}(X, \mathbb{Q})$.

Given any rational number $\alpha \in \mathbb{Q}$, define the ring

$$
\mathrm{QH}_{\alpha}=S[q] /\left\langle\sigma_{k+1}, \ldots, \sigma_{n-1}, \sigma_{n}+(-1)^{m} \alpha q\right\rangle \text {. }
$$

This ring is a graded $\mathbb{Q}[q]$-algebra, where the grading is defined by $\operatorname{deg}(q)=n$ and $\operatorname{deg}\left(c_{p}\right)=p$ for $1 \leq p \leq m$. Lemma 12 implies that the assignments $\sigma_{\lambda} \mapsto\left[X^{\lambda}\right]$ and $q \mapsto 0$ define a ring homomorphism $\mathrm{QH}_{\alpha} \rightarrow H^{*}(X, \mathbb{Q})$, and we show in Corollary 14 that $\mathrm{QH}_{\alpha}$ is a quantum deformation of $H^{*}(X, \mathbb{Q})$ with basis $\left\{\sigma_{\lambda} \mid \lambda \subset m \times k\right\}$. We first show that $\mathrm{QH}_{\alpha}$ satisfies the following rescaled version of Bertram's quantum Pieri formula [4]. 
Proposition 13. (a) For $1 \leq p \leq m$ and $\lambda \subset m \times k$ we have in $\mathrm{QH}_{\alpha}$ that

$$
c_{p} \sigma_{\lambda}=\sum_{\mu} \sigma_{\mu}+\sum_{\nu} \alpha q \sigma_{\nu}
$$

The first sum is over all partitions $\mu \subset m \times k$ that can be obtained by adding a vertical strip of $p$ boxes to $\lambda$. The second sum is empty unless $\lambda_{1}=k$, in which case it is over all partitions $\nu$ obtained by removing $n-p$ boxes from the outer rim of $\lambda$, with at least one box removed from each column.

(b) For $1 \leq p \leq k$ and $\lambda \subset m \times k$ we have in $\mathrm{QH}_{\alpha}$ that

$$
\sigma_{p} \sigma_{\lambda}=\sum_{\mu} \sigma_{\mu}+\sum_{\nu} \alpha q \sigma_{\nu}
$$

The first sum is over all partitions $\mu \subset m \times k$ that can be obtained by adding a horizontal strip of $p$ boxes to $\lambda$. The second sum is empty unless $\lambda_{m} \neq 0$, in which case it is over all partitions $\nu$ obtained by removing $n-p$ boxes from the outer rim of $\lambda$, with at least one box removed from each row.

Proof. It follows from Fact 7 and Lemma 9 that

$$
c_{p} \sigma_{\lambda}=\sum_{\mu} \sigma_{\mu}
$$

where the sum is over all partitions $\mu$ with at most $m$ rows that can be obtained by adding a vertical strip of size $p$ to $\lambda$. If such a partition satisfies $\mu_{1} \leq k$, then the term $\sigma_{\mu}$ is included in the first sum of part (a). Otherwise we have $\lambda_{1}=k$ and $\mu_{1}=k+1$, in which case the first row of the $m \times m$ determinant defining $\sigma_{\mu}$ is equal to $\left(0, \ldots, 0,(-1)^{m-1} \alpha q\right)$ modulo the ideal defining $\mathrm{QH}_{\alpha}$. If $\mu_{m}=0$, then the last row of the same determinant is $(0, \ldots, 0,1)$, in which case $\sigma_{\mu}=0$. On the other hand, if $\mu_{m}>0$, then $\sigma_{\mu}=\alpha q \sigma_{\nu} \in \mathrm{QH}_{\alpha}$ where $\nu=\left(\mu_{2}-1, \ldots, \mu_{m}-1,0\right)$. The second sum of part (a) contains exactly the terms obtained in this way.

Part (b) is equivalent to part (a) with $m$ and $k$ interchanged. To make this explicit, let $c^{\prime}=\left(c_{1}^{\prime}, \ldots, c_{k}^{\prime}, 0,0, \ldots\right)$ be a sequence of $k$ variables, and set $\sigma_{p}^{\prime}=$ $\Delta_{\left(1^{p}\right)}\left(c^{\prime}\right)$ for $p \geq 0$ and $\sigma_{\mu}^{\prime}=\Delta_{\mu}\left(\sigma^{\prime}\right)$ for $\mu \subset k \times m$. Let $\phi: \mathbb{Q}\left[c_{1}^{\prime}, \ldots, c_{k}^{\prime}\right] \rightarrow$ $\mathbb{Q}\left[c_{1}, \ldots, c_{m}\right]$ be the ring homomorphism defined by $\phi\left(c_{j}^{\prime}\right)=\sigma_{j}$ for $1 \leq j \leq k$. For $1 \leq p<n$ we have $\phi\left(\sigma_{p}^{\prime}\right)=\Delta_{\left(1^{p}\right)}\left(\sigma_{1}, \ldots, \sigma_{k}, 0, \ldots\right) \equiv \Delta_{\left(1^{p}\right)}(\sigma)=c_{p}$ modulo the ideal $J=\left\langle\sigma_{k+1}, \ldots, \sigma_{n-1}\right\rangle$. This implies that $\phi\left(\sigma_{\mu}^{\prime}\right) \equiv \Delta_{\mu}(c)=\Delta_{\mu^{\prime}}(\sigma)=\sigma_{\mu^{\prime}}(\bmod$ $J)$ for $\mu \subset k \times m$. We also have $\phi\left(\sigma_{n}^{\prime}\right)=\Delta_{\left(1^{n}\right)}\left(\sigma_{1}, \ldots, \sigma_{k}, 0, \ldots\right) \equiv \Delta_{\left(1^{n}\right)}(\sigma)+$ $(-1)^{n} \sigma_{n}=(-1)^{n} \sigma_{n}(\bmod J)$; this term appears because $\sigma_{n}$ is the upper-right entry of the determinant defining $\Delta_{\left(1^{n}\right)}(\sigma)$. Define

$$
\mathrm{QH}_{\alpha}^{\prime}=\mathbb{Q}\left[q, c_{1}^{\prime}, \ldots, c_{k}^{\prime}\right] /\left\langle\sigma_{m+1}^{\prime}, \ldots, \sigma_{n-1}^{\prime}, \sigma_{n}^{\prime}+(-1)^{k} \alpha q\right\rangle .
$$

The observed properties of $\phi$ show that this map factors as a $\mathbb{Q}[q]$-algebra homomorphism $\phi: \mathrm{QH}_{\alpha}^{\prime} \rightarrow \mathrm{QH}_{\alpha}$ given by $\phi\left(\sigma_{\mu}^{\prime}\right)=\sigma_{\mu^{\prime}}$ for $\mu \subset k \times m$. Part (b) of the proposition therefore follows from part (a) applied to $\mathrm{QH}_{\alpha}^{\prime}$.

Corollary 14. $\mathrm{QH}_{\alpha}$ is a free $\mathbb{Q}[q]$-module with basis $\left\{\sigma_{\lambda} \mid \lambda \subset m \times k\right\}$.

Proof. It follows from Proposition $13(\mathrm{a})$ that $\mathrm{QH}_{\alpha}$ is generated as a $\mathbb{Q}[q]$-module by the classes $\sigma_{\lambda}$ for $\lambda \subset m \times k$, and Corollary 11 implies that $q$ is a non-zero divisor in $\mathrm{QH}_{\alpha}$. Assume that $\sum_{\lambda} a_{\lambda} \sigma_{\lambda}=0$ is a non-trivial relation in $\mathrm{QH}_{\alpha}$, where the sum is over $\lambda \subset m \times k$ and $a_{\lambda} \in \mathbb{Q}[q]$. Since $q$ is a non-zero divisor, we may assume 
that $a_{\lambda}(0) \neq 0$ for some $\lambda$. But then the relation $\sum_{\lambda} a_{\lambda}(0) \sigma_{\lambda}=0$ in $\mathrm{QH}_{\alpha} /\langle q\rangle$ contradicts Lemma 10.

\subsection{The Seidel representation.}

Definition 15. Given a partition $\lambda \subset m \times k$, define the first Seidel shift of $\lambda$ by

$$
\lambda \uparrow 1= \begin{cases}\left(\lambda_{1}+1, \lambda_{2}+1, \ldots, \lambda_{m}+1\right) & \text { if } \lambda_{1}<k, \\ \left(\lambda_{2}, \lambda_{3}, \ldots, \lambda_{m}, 0\right) & \text { if } \lambda_{1}=k .\end{cases}
$$

The $p$-th Seidel shift $\lambda \uparrow p$ is defined inductively by $\lambda \uparrow 0=\lambda$ and $\lambda \uparrow(p+1)=$ $(\lambda \uparrow p) \uparrow 1$ for $p \geq 0$.

The first Seidel shift captures the following case of Bertram's quantum Pieri formula (Proposition 13):

$$
c_{m} \sigma_{\lambda}=(\alpha q)^{\frac{m+|\lambda|-|\lambda \uparrow 1|}{n}} \sigma_{\lambda \uparrow 1} .
$$

Notice that Seidel shifts of the zero partition are given by

$$
0 \uparrow p= \begin{cases}\left(p^{m}\right) & \text { for } 0 \leq p \leq k, \\ \left(k^{n-p}\right) & \text { for } k \leq p \leq n .\end{cases}
$$

It follows that $c_{m}^{n}=(\alpha q)^{m}$ and $(\alpha q)^{m} \sigma_{\lambda}=c_{m}^{n} \sigma_{\lambda}=(\alpha q)^{\frac{m n+|\lambda|-|\lambda \uparrow n|}{n}} \sigma_{\lambda \uparrow n}$, so we obtain $\lambda \uparrow n=\lambda$ for any partition $\lambda \subset m \times k$. For any integer $p \in \mathbb{Z}$ we can therefore define $\lambda \uparrow p=\lambda \uparrow p^{\prime}$ where $p^{\prime} \geq 0$ is chosen so that $p^{\prime} \equiv p(\bmod n)$. We write $\lambda \downarrow p=\lambda \uparrow(-p)$ for convenience. With this notation we have

$$
\sigma_{k} \sigma_{\lambda}=(\alpha q)^{\frac{k+|\lambda|-|\lambda \downarrow 1|}{n}} \sigma_{\lambda \downarrow 1}
$$

in $\mathrm{QH}_{\alpha}$ by Proposition 13(b).

Lemma 16. For any partition $\lambda \subset m \times k$ we have $\sum_{p=0}^{n-1}|\lambda \uparrow p|=\frac{1}{2} k m n$.

Proof. The lemma follows from (3) when $\lambda=0$, so it suffices to show that $\sum_{p=0}^{n-1} \mid \mu \uparrow$ $p\left|=\sum_{p=0}^{n-1}\right| \lambda \uparrow p \mid$ holds whenever the partition $\mu$ is obtained by adding a single box to $\lambda$, say in row $i$ and column $j$. By replacing the partitions with $\lambda \uparrow(k-j+i-1)$ and $\mu \uparrow(k-j+i-1)$, we may assume that the added box is in the upper-right corner of the rectangle $m \times k$, so that $\lambda_{1}=k-1$ and $\mu_{1}=k$. The identity then follows because $|\mu \downarrow p|=|\lambda \downarrow p|+1$ for $0 \leq p \leq n-2$ and $|\mu \uparrow 1|=|\lambda \uparrow 1|-n+1$.

Corollary 17. Let $\lambda$ and $\mu$ be partitions contained in $m \times k$ such that $|\lambda|>|\mu|$. Then there exists $p \in \mathbb{Z}$ for which $|\lambda \uparrow p|<|\mu \uparrow p|$.

3.6. Main results. Our classification of non-negative quantum deformations of Grassmannians is based on the following theorem.

Theorem 18. Let $\left\{\tau_{\lambda} \mid \lambda \subset m \times k\right\}$ be any $\mathbb{Q}[q]$-basis of $\mathrm{QH}_{\alpha}$ such that (1) $\tau_{\lambda}$ is homogeneous of degree $|\lambda|$, (2) $\tau_{\lambda}-\sigma_{\lambda} \in\langle q\rangle$, and (3) the structure constants of $\mathrm{QH}_{\alpha}$ with respect to the $\mathbb{Q}$-basis $\left\{q^{d} \tau_{\lambda} \mid \lambda \subset m \times k, d \geq 0\right\}$ are non-negative. Then we have $\tau_{\lambda}=\sigma_{\lambda}$ for all $\lambda$.

Proof. For each $\lambda \subset m \times k$ it follows from (1) and (2) that we can write

$$
\tau_{\lambda}=\sigma_{\lambda}+\sum_{|\mu|<|\lambda|} a_{\lambda, \mu} q^{\frac{|\lambda|-|\mu|}{n}} \sigma_{\mu}
$$


where $a_{\lambda, \mu} \in \mathbb{Q}$ is non-zero only if $|\lambda|-|\mu|$ is a positive multiple of $n$. We must show that $a_{\lambda, \mu}=0$ for all $\lambda$ and $\mu$. Since $c_{m} \tau_{k}=\alpha q$, we must have $\alpha \geq 0$ by (3).

Assume first that $\alpha=0$. If the theorem is false, then let $\lambda \subset m \times k$ be minimal in the degree-lexicographic order such that $\tau_{\lambda} \neq \sigma_{\lambda}$. Set $\ell=\ell(\lambda)$ and let $\widehat{\lambda}=$ $\left(\lambda_{1}-1, \lambda_{2}-1, \ldots, \lambda_{\ell}-1\right)$ be the partition obtained by removing the first column of $\lambda$. Proposition 13(a) shows that

$$
c_{\ell} \tau_{\widehat{\lambda}}=c_{\ell} \sigma_{\widehat{\lambda}}=\sum_{\nu} \sigma_{\nu}
$$

where the sum is over all partitions $\nu \subset m \times k$ that can be obtained by adding a vertical strip of $\ell$ boxes to $\widehat{\lambda}$. Since $\lambda$ is the maximal such partition in the degreelexicographic order, we obtain

$$
c_{\ell} \tau_{\widehat{\lambda}}=\sigma_{\lambda}+\sum_{\nu \neq \lambda} \sigma_{\nu}=\tau_{\lambda}-\sum_{|\mu|<|\lambda|} a_{\lambda, \mu} q^{\frac{|\lambda|-|\mu|}{n}} \tau_{\mu}+\sum_{\nu \neq \lambda} \tau_{\nu} .
$$

This shows that $-a_{\lambda, \mu}$ is a structure constant of the basis $\mathcal{T}=\left\{q^{d} \tau_{\lambda}\right\}$ for each $\mu$, so (3) implies that $a_{\lambda, \mu} \leq 0$. Now choose $\nu$ with $a_{\lambda, \nu}<0$ such that the dual partition $\nu^{\vee}=\left(k-\nu_{m}, \ldots, k-\nu_{1}\right)$ is maximal in the degree-lexicographic order. If $\mu$ is any partition for which $a_{\lambda, \mu} \neq 0$, then either $\nu^{\vee}$ is larger than $\mu^{\vee}$ in the lexicographic order, in which case it follows from Proposition 13(b) and induction on $p$ that

$$
\sigma_{\nu_{1}^{\vee}} \cdots \sigma_{\nu_{p}^{\vee}} \sigma_{\mu}= \begin{cases}\sigma_{\left(k^{p}, \mu_{1}, \ldots, \mu_{m-p}\right)} & \text { if } \mu_{i}=\nu_{i} \text { for } m-p<i \leq m \\ 0 & \text { otherwise. }\end{cases}
$$

Otherwise we have $\left|\nu^{\vee}\right|>\left|\mu^{\vee}\right|$, in which case $\left|\nu^{\vee}\right|+|\mu|>m k$ and $\sigma_{\nu_{1}^{\vee}} \cdots \sigma_{\nu_{m}^{\vee}} \sigma_{\mu}=0$.

Applying this to equation (5) we therefore obtain

$$
\tau_{\nu_{1}^{\vee}} \cdots \tau_{\nu_{m}^{\vee}} \tau_{\lambda}=a_{\lambda, \nu} q^{\frac{|\lambda|-|\nu|}{n}} \sigma_{\left(k^{m}\right)}
$$

Finally, since the coefficient of $\tau_{\left(k^{m}\right)}$ in the $\mathcal{T}$-expansion of $\sigma_{\left(k^{m}\right)}$ is equal to 1 , we deduce that $a_{\lambda, \nu}$ is an iterated structure constant, so (3) implies that $a_{\lambda, \nu} \geq 0$, a contradiction.

Assume now that $\alpha>0$. Then $c_{m} \tau_{k}=\alpha q$ is a non-zero multiple of $q$. If $\lambda \subset m \times k$ is any partition with $\lambda_{1}<k$, then since $\left[X^{\left(1^{m}\right)}\right] \cdot\left[X^{\lambda}\right]=\left[X^{\lambda \uparrow 1}\right]$ holds in $H^{*}(X, \mathbb{Q})$, it follows from Lemma 3 that $c_{m} \tau_{\lambda}=\tau_{\lambda \uparrow 1}$. On the other hand, if $\lambda_{1}=k$, then $\left[X^{k}\right] \cdot\left[X^{\lambda \uparrow 1}\right]=\left[X^{\lambda}\right]$ holds in $H^{*}(X, \mathbb{Q})$, so Lemma 3 implies that $c_{m} \tau_{\lambda}=c_{m} \tau_{k} \tau_{\lambda \uparrow 1}=\alpha q \tau_{\lambda \uparrow 1}$. This shows that multiplication by $c_{m}$ in the basis $\mathcal{T}$ is determined by

$$
c_{m} \tau_{\lambda}=(\alpha q)^{\frac{m+|\lambda|-|\lambda \uparrow 1|}{n}} \tau_{\lambda \uparrow 1}
$$

If $\tau_{\lambda} \neq \sigma_{\lambda}$, then choose $\mu$ such that $a_{\lambda, \mu} \neq 0$. By Corollary 17 we can choose $p$ such that $|\lambda \uparrow p|<|\mu \uparrow p|$. Equation (6) then implies that $c_{m}^{p} \tau_{\lambda}=(\alpha q)^{d} \tau_{\lambda \uparrow p}$ where $d=\frac{m p+|\lambda|-|\lambda \uparrow p|}{n}$, so $c_{m}^{p} \tau_{\lambda}$ is divisible by $q^{d}$. However, equation (2) shows that the product of $c_{m}^{p}$ with the right hand side of (5) contains the term

$$
a_{\lambda, \mu} q^{\frac{|\lambda|-|\mu|}{n}}(\alpha q)^{\frac{m p+|\mu|-|\mu \uparrow p|}{n}} \sigma_{\mu \uparrow p}
$$

and since $|\lambda \uparrow p|<|\mu \uparrow p|$, this term is not divisible by $q^{d}$. This contradicts that $c_{m}^{p} \tau_{\lambda}$ is divisible by $q^{d}$, which completes the proof. 
Corollary 19. Let $X=\operatorname{Gr}(m, n)$ and let $\mathrm{QH}_{\alpha}=\left(\mathrm{QH}_{\alpha},\left\{\sigma_{\lambda}\right\}\right)$ be the quantum deformation of $H^{*}(X, \mathbb{Q})$ defined in Section 3.4.

(a) The deformation $\mathrm{QH}_{\alpha}$ is non-negative if and only if $\alpha \geq 0$.

(b) Any non-negative quantum deformation of $H^{*}(X, \mathbb{Q})$ is isomorphic to $\mathrm{QH}_{\alpha}$ for a unique rational number $\alpha \geq 0$.

(c) $\mathrm{QH}(X) \cong \mathrm{QH}_{1}$ is the only non-negative change-of-basis quantum deformation of $H^{*}(X, \mathbb{Q})$.

Proof. Given any quantum deformation $\left(\mathrm{QH},\left\{\tau_{\lambda}\right\}\right)$ of $H^{*}(X, \mathbb{Q})$, it follows from Definition 2 that $\tau_{\left(1^{m}\right)} \tau_{k}=\alpha q$ for some $\alpha \in \mathbb{Q}$. Define a ring homomorphism $\phi$ : $S[q] \rightarrow \mathrm{QH}$ by $\phi\left(c_{p}\right)=\tau_{\left(1^{p}\right)}$ and $\phi(q)=q$. Definition 2 then implies that $\phi\left(\sigma_{p}\right)=\tau_{p}$ for $1 \leq p \leq k, \phi\left(\sigma_{p}\right)=0$ for $k<p<n$, and $\phi\left(\sigma_{n}\right)=\phi\left(\sum_{p=1}^{m}(-1)^{p-1} c_{p} \sigma_{n-p}\right)=$ $(-1)^{m-1} \tau_{\left(1^{m}\right)} \tau_{k}=(-1)^{m-1} \alpha q$. We deduce that $\phi$ factors as an isomorphism of $\mathbb{Q}[q]$-algebras $\psi: \mathrm{QH}_{\alpha} \rightarrow \mathrm{QH}$, and the set $\left\{\psi^{-1}\left(\tau_{\lambda}\right)\right\}$ is a deformation of the basis $\left\{\sigma_{\lambda}\right\}$ of $\mathrm{QH}_{\alpha}$. If $\mathrm{QH}$ is a non-negative quantum deformation, then Theorem 18 implies that $\psi^{-1}\left(\tau_{\lambda}\right)=\sigma_{\lambda}$ for each $\lambda$. This proves part (b).

Part (c) follows from part (b) by using that $\mathrm{QH}(X)$ is a non-negative quantum deformation of $H^{*}(X, \mathbb{Q})$ satisfying the relation $\left[X^{\left(1^{m}\right)}\right] \star\left[X^{k}\right]=q$. This relation is equivalent to Witten's presentation of $\mathrm{QH}(X)[54, \S 3.2]$ (see also [53, Prop. 3.1]). The uniqueness statement of part (c) follows from Theorem 18 applied to $\mathrm{QH}_{1}$.

For $\alpha>0$ the assignments $q \mapsto \alpha q$ and $c_{p} \mapsto c_{p}$ define an isomorphism of graded rings $\mathrm{QH}_{1} \cong \mathrm{QH}_{\alpha}$. Since this map sends $\sigma_{\lambda}$ to $\sigma_{\lambda}$, it follows that the structure constants of $\mathrm{QH}_{\alpha}$ are the rescaled Gromov-Witten invariants $\alpha^{d}\left\langle\left[X^{\lambda}\right],\left[X^{\mu}\right],\left[X_{\nu}\right]\right\rangle_{d}$. This shows that $\mathrm{QH}_{\alpha}$ is non-negative for $\alpha>0$. The trivial deformation $\mathrm{QH}_{0}=$ $H^{*}(X, \mathbb{Q}) \otimes_{\mathbb{Q}} \mathbb{Q}[q]$ is also non-negative, and the relation $c_{m} \sigma_{k}=\alpha q$ shows that $\mathrm{QH}_{\alpha}$ is not non-negative for $\alpha<0$. This proves part (a).

Corollary 20. The quantum cohomology ring $\mathrm{QH}(X)$ satisfies Bertram's quantum Giambelli formula

$$
\left[X^{\lambda}\right]=\operatorname{det}\left(\left[X^{\lambda_{i}+j-i}\right]\right)_{m \times m}
$$

and quantum Pieri formula

$$
\left[X^{p}\right] \star\left[X^{\lambda}\right]=\sum_{\mu}\left[X^{\mu}\right]+\sum_{\nu} q\left[X^{\nu}\right]
$$

where the sums are over the same partitions as in Proposition 13(b).

Proof. This follows from Corollary 19, Proposition 13, and the definition of $\sigma_{\lambda}$.

Example 21. Let $X=\mathrm{GL}(6) / B$ be the variety of complete flags in $\mathbb{C}^{6}$ and set $t=(6,1,2,3,4,5)$. We have $\pi_{1}(\operatorname{Aut}(X)) \cong \mathbb{Z} / 6 \mathbb{Z}$, and the Seidel representation of this group on $\mathrm{QH}(X) /\langle q-1\rangle$ is defined by quantum multiplication with $\left[X^{t}\right]$, see $[52,19]$. Products with this class modulo $\langle q-1\rangle$ are given by $\left[X^{t}\right] \star\left[X^{w}\right]=\left[X^{t w}\right]$ [21]. The following table shows the Seidel orbits of the Schubert classes given by $e=(1,2,3,4,5,6)$ and $v=(3,2,1,6,5,4)$, using the notation $w \uparrow r=t^{r} w$. 


\begin{tabular}{|c|c|c|c|c|}
\hline$r$ & $e \uparrow r$ & $\ell(e \uparrow r)$ & $v \uparrow r$ & $\ell(v \uparrow r)$ \\
\hline 0 & 123456 & 0 & 321654 & 6 \\
1 & 612345 & 5 & 216543 & 7 \\
2 & 561234 & 8 & 165432 & 10 \\
3 & 456123 & 9 & 654321 & 15 \\
4 & 345612 & 8 & 543216 & 10 \\
5 & 234561 & 5 & 432165 & 7 \\
\hline
\end{tabular}

This shows that the analogues of Lemma 16 and Corollary 17 for varieties of complete flags are false.

\section{REFERENCES}

[1] D. Anderson and L. Chen, Equivariant quantum Schubert polynomials, Adv. Math. 254 (2014), 300-330. MR 3161101

[2] _ Positivity of equivariant Gromov-Witten invariants, Math. Res. Lett. 22 (2015), no. 1, 1-9. MR 3342175

[3] D. Anderson, L. Chen, and H.-H. Tseng, The quantum K-theory of a homogeneous space is finite, arXiv:1804.04579.

[4] A. Bertram, Quantum Schubert calculus, Adv. Math. 128 (1997), no. 2, 289-305. MR 1454400 (98j:14067)

[5] A. Bertram, I. Ciocan-Fontanine, and W. Fulton, Quantum multiplication of Schur polynomials, J. Algebra 219 (1999), no. 2, 728-746. MR 1706853 (2000k:14042)

[6] A. S. Buch, Quantum cohomology of Grassmannians, Compositio Math. 137 (2003), no. 2, 227-235. MR 1985005

[7] _ Mutations of puzzles and equivariant cohomology of two-step flag varieties, Ann. of Math. (2) 182 (2015), no. 1, 173-220. MR 3374959

[8] A. S. Buch, P.-E. Chaput, L. Mihalcea, and N. Perrin, A Chevalley formula for the equivariant quantum $K$-theory of cominuscule varieties, arXiv:1604.07500, to appear in Algebraic Geometry.

[9] _ Finiteness of cominuscule quantum K-theory, Ann. Sci. Éc. Norm. Supér. (4) 46 (2013), no. 3, 477-494 (2013). MR 3099983

[10] - Projected Gromov-Witten varieties in cominuscule spaces, Proc. Amer. Math. Soc. 146 (2018), no. 9, 3647-3660. MR 3825822

[11] A. S. Buch, S. Chung, C. Li, and C. Mihalcea, Euler characteristics in the quantum K-theory of flag varieties., arXiv:1903.02215.

[12] A. S. Buch, A. Kresch, K. Purbhoo, and H. Tamvakis, The puzzle conjecture for the cohomology of two-step flag manifolds, J. Algebraic Combin. 44 (2016), no. 4, 973-1007. MR 3566227

[13] A. S. Buch, A. Kresch, and H. Tamvakis, Gromov-Witten invariants on Grassmannians, J. Amer. Math. Soc. 16 (2003), no. 4, 901-915. MR 1992829

[14] _ Quantum Pieri rules for isotropic Grassmannians, Invent. Math. 178 (2009), no. 2, 345-405. MR 2545685

[15] — A Giambelli formula for even orthogonal Grassmannians, J. Reine Angew. Math. 708 (2015), 17-48. MR 3420327

[16] _ A Giambelli formula for isotropic Grassmannians, Selecta Math. (N.S.) 23 (2017), no. 2, 869-914. MR 3624902

[17] A. S. Buch and L. Mihalcea, Quantum K-theory of Grassmannians, Duke Math. J. 156 (2011), no. 3, 501-538. MR 2772069

[18] P.-E. Chaput, L. Manivel, and N. Perrin, Quantum cohomology of minuscule homogeneous spaces, Transform. Groups 13 (2008), no. 1, 47-89. MR 2421317 (2009e:14095)

[19] _ Affine symmetries of the equivariant quantum cohomology ring of rational homogeneous spaces, Math. Res. Lett. 16 (2009), no. 1, 7-21. MR 2480556 (2010h:14086)

[20] I. Ciocan-Fontanine, Quantum cohomology of flag varieties, Internat. Math. Res. Notices (1995), no. 6, 263-277. MR 1344348 (96h:14071)

[21] - On quantum cohomology rings of partial flag varieties, Duke Math. J. 98 (1999), no. 3, 485-524. MR 1695799 (2000d:14058) 
[22] S. Fomin, S. Gelfand, and A. Postnikov, Quantum Schubert polynomials, J. Amer. Math. Soc. 10 (1997), no. 3, 565-596. MR 1431829 (98d:14063)

[23] W. Fulton, Young tableaux, London Mathematical Society Student Texts, vol. 35, Cambridge University Press, Cambridge, 1997, With applications to representation theory and geometry. MR 1464693

[24] , private communication, 2001.

[25] W. Fulton and R. Pandharipande, Notes on stable maps and quantum cohomology, Algebraic geometry-Santa Cruz 1995, Proc. Sympos. Pure Math., vol. 62, Amer. Math. Soc., Providence, RI, 1997, pp. 45-96. MR 1492534

[26] W. Fulton and C. Woodward, On the quantum product of Schubert classes, J. Algebraic Geom. 13 (2004), no. 4, 641-661. MR 2072765

[27] A. Givental, On the WDVV equation in quantum K-theory, Michigan Math. J. 48 (2000), 295-304, Dedicated to William Fulton on the occasion of his 60th birthday. MR 1786492 (2001m:14078)

[28] A. Givental and B. Kim, Quantum cohomology of flag manifolds and Toda lattices, Comm. Math. Phys. 168 (1995), no. 3, 609-641. MR 1328256 (96c:58027)

[29] V. Gorbounov and C. Korff, Quantum integrability and generalised quantum Schubert calculus, Adv. Math. 313 (2017), 282-356. MR 3649227

[30] H. Hiller and B. Boe, Pieri formula for $\mathrm{SO}_{2 n+1} / \mathrm{U}_{n}$ and $\mathrm{Sp}_{n} / \mathrm{U}_{n}$, Adv. in Math. 62 (1986), no. 1, 49-67. MR 859253 (87k:14058)

[31] Y. Huang and C. Li, On equivariant quantum Schubert calculus for $G / P$, J. Algebra 441 (2015), 21-56. MR 3391918

[32] H. Iritani, T. Milanov, and V. Tonita, Reconstruction and convergence in quantum K-theory via difference equations, Int. Math. Res. Not. IMRN (2015), no. 11, 2887-2937. MR 3373040

[33] S. Kato, Frobenius splitting of Schubert varieties of semi-infinite flag manifolds, arXiv:1810.07106.

[34] L Loop structure on equivariant $K$-theory of semi-infinite flag manifolds, arXiv:1805.01718.

[35] B. Kim, Quantum cohomology of partial flag manifolds and a residue formula for their intersection pairings, Internat. Math. Res. Notices (1995), no. 1, 1-15 (electronic). MR 1317639 (96c:58028)

[36] On equivariant quantum cohomology, Internat. Math. Res. Notices (1996), no. 17, 841-851. MR 1420551 (98h:14013)

[37] Luantum cohomology of flag manifolds $G / B$ and quantum Toda lattices, Ann. of Math. (2) 149 (1999), no. 1, 129-148. MR 1680543 (2001c:14081)

[38] M. Kontsevich and Yu. Manin, Gromov-Witten classes, quantum cohomology, and enumerative geometry, Comm. Math. Phys. 164 (1994), no. 3, 525-562. MR 1291244 (95i:14049)

[39] A. Kresch and H. Tamvakis, Quantum cohomology of the Lagrangian Grassmannian, J. Algebraic Geom. 12 (2003), no. 4, 777-810. MR 1993764 (2004h:14063)

[40] _ Quantum cohomology of orthogonal Grassmannians, Compos. Math. 140 (2004), no. 2, 482-500. MR 2027200 (2005d:14080)

[41] T. Lam, C. Li, L. Mihalcea, and M. Shimozono, A conjectural Peterson isomorphism in K-theory, J. Algebra 513 (2018), 326-343. MR 3849889

[42] Y.-P. Lee, Quantum K-theory. I. Foundations, Duke Math. J. 121 (2004), no. 3, 389-424. MR 2040281 (2005f:14107)

[43] C. Lenart and T. Maeno, Quantum Grothendieck polynomials, math.CO/0608232.

[44] C. Lenart and A. Postnikov, Affine Weyl groups in K-theory and representation theory, Int. Math. Res. Not. IMRN (2007), no. 12, Art. ID rnm038, 65. MR 2344548

[45] I. G. Macdonald, Symmetric functions and Hall polynomials, second ed., Oxford Mathematical Monographs, The Clarendon Press Oxford University Press, New York, 1995, With contributions by A. Zelevinsky, Oxford Science Publications. MR 1354144 (96h:05207)

[46] L. Manivel, Symmetric functions, Schubert polynomials and degeneracy loci, SMF/AMS Texts and Monographs, vol. 6, American Mathematical Society, Providence, RI, 2001, Translated from the 1998 French original by John R. Swallow, Cours Spécialisés [Specialized Courses], 3. MR 1852463 (2002h:05161)

[47] H. Matsumura, Commutative ring theory, second ed., Cambridge Studies in Advanced Mathematics, vol. 8, Cambridge University Press, Cambridge, 1989, Translated from the Japanese by M. Reid. MR 1011461 
[48] L. Mihalcea, Positivity in equivariant quantum Schubert calculus, Amer. J. Math. 128 (2006), no. 3, 787-803. MR 2230925

[49] - On equivariant quantum cohomology of homogeneous spaces: Chevalley formulae and algorithms, Duke Math. J. 140 (2007), no. 2, 321-350. MR 2359822

[50] Giambelli formulae for the equivariant quantum cohomology of the Grassmannian, Trans. Amer. Math. Soc. 360 (2008), no. 5, 2285-2301. MR 2373314

[51] Y. Ruan and G. Tian, A mathematical theory of quantum cohomology, Math. Res. Lett. 1 (1994), no. 2, 269-278. MR 1266766 (95b:58025)

[52] P. Seidel, $\pi_{1}$ of symplectic automorphism groups and invertibles in quantum homology rings, Geom. Funct. Anal. 7 (1997), no. 6, 1046-1095. MR 1487754

[53] B. Siebert and G. Tian, On quantum cohomology rings of Fano manifolds and a formula of Vafa and Intriligator, Asian J. Math. 1 (1997), no. 4, 679-695. MR 1621570 (99d:14060)

[54] E. Witten, The Verlinde algebra and the cohomology of the Grassmannian, Geometry, topology, \& physics, Conf. Proc. Lecture Notes Geom. Topology, IV, Int. Press, Cambridge, MA, 1995, pp. 357-422. MR 1358625 (98c:58016)

[55] C. T. Woodward, On D. Peterson's comparison formula for Gromov-Witten invariants of G/P, Proc. Amer. Math. Soc. 133 (2005), no. 6, 1601-1609. MR 2120266

Department of Mathematics, Rutgers University, 110 Frelinghuysen Road, PiscatAWAY, NJ 08854, USA

E-mail address: asbuch@math.rutgers.edu

Department of Mathematics, Rutgers University, 110 Frelinghuysen Road, PiscatAWAY, NJ 08854, USA

E-mail address: cw674@math.rutgers.edu 